\title{
OXIDATION STUDIES \\ OF THE GRAPHITE SURFACE BY SCANNING TUNNELING MICROSCOPY AND PHOTOELECTRON SPECTROSCOPY
}

\author{
Z. Klusek*, M. Phaner, P. Krapf and L. Porte \\ Ecole Centrale de Lyon, B.P. 163, 69131 Ecully Cx, France
}

(Received July 5, 1994)

\begin{abstract}
Scanning tunneling microscopy and spectroscopy and X-ray photoelectron spectroscopy were used to study oxidation effects of nitric acid on highly oriented pyrolytic graphite surface. This treatment was performed at different temperatures and etching times with the aim of realizing local binding sites on the surface without creating deep defects. Then these three technics were found in good agreement to characterize the weakly oxidized surface. A wavy structure different from pure graphite at atomic scale was imaged by scanning tunneling microscopy. This modification was correlated to the presence of carboxylic groups on the surface revealed by X-ray photoelectron spectroscopy. Both spectroscopies of scanning tunneling and X-ray photoelectron demonstrated the vanishing of $\pi$ bands characteristic from $s p^{2}$ graphitic hybridization. This was attributed to dehybridization corresponding to new bondings of the graphite carbons in the carboxylic groups.
\end{abstract}

PACS numbers: $61.16 . \mathrm{Ch}, 33.60 . \mathrm{Fy}$

\section{Introduction}

Creating binding sites homogeneously distributed on the basal plane of a layered material such as graphite substrate can be of considerable interest for deposited aggregates or molecular species. It can be useful not only for the chemical physicist to study the distribution of metallic catalysts chemisorbed on carbon substrate but for the biologist too to fix biopolymers on solid supports and determine their structure. It is well known that these reactive sites can be obtained by an oxidation treatment of the surface. However, most of the previous studies $[1,2]$

${ }^{*}$ On lea ve from the Department of Solid State Physics, University of Łódź, Pomorska 149/153, 90-236 Łódź, Poland. 
showed deep defects (pits or holes) as etching effects randomly distributed on the surface.

In this paper a smooth oxidation treatment due to nitric acid at different temperatures and time of etching is proposed. The characterization of the oxidized surface is performed in comparison with pure graphite by the combination of two complementary technics: X-ray photoelectron spectroscopy (XPS) and scanning tunneling microscopy/spectroscopy (STM/STS). XPS allows a global and precise identification of the chemical compounds of the surface and STM as a local high resolution technic presents images of the atomic modification of the graphitic lattice. Scanning tunneling spectroscopy is also used to confirm the quite different electronic behavior of oxidized and pure graphite.

\section{Experiment}

A new surface of highly oriented pyrolytic graphite (HOPG) was prepared by cleavage with adhesive tape before the block of graphite was deeped in a $4 \mathrm{~N}$ solution of nitric acid. This is a standard procedure to create active sites on the graphite surface for catalytic purpose [1, 3, 4]. Two different etching temperatures $\left(20^{\circ} \mathrm{C}\right.$ and $\left.70^{\circ} \mathrm{C}\right)$ and different etching times were used. After the acid treatment the samples were rinsed with distilled water then dried for 30 minutes at $140^{\circ} \mathrm{C}$. Table gives the treatment of different samples.

TABLE

Presentation of five different oxidized HOPG samples with their preparation conditions.

\begin{tabular}{c|cc|c|c}
\hline \hline Sample & Solution & Temperature & Etching time \\
\hline$A$ & $4 \mathrm{~N}$ & $\mathrm{HNO}_{3}$ & $70^{\circ} \mathrm{C}$ & $5 \mathrm{~min}$ \\
$B$ & $4 \mathrm{~N}$ & $\mathrm{HNO}_{3}$ & $70^{\circ} \mathrm{C}$ & $10 \mathrm{~min}$ \\
$C$ & $4 \mathrm{~N}$ & $\mathrm{HNO}_{3}$ & $70^{\circ} \mathrm{C}$ & $20 \mathrm{~min}$ \\
$D$ & $4 \mathrm{~N}$ & $\mathrm{HNO}_{3}$ & $70^{\circ} \mathrm{C}$ & $30 \mathrm{~min}$ \\
$E$ & $4 \mathrm{~N}$ & $\mathrm{HNO}_{3}$ & $20^{\circ} \mathrm{C}$ & $30 \mathrm{~min}$
\end{tabular}

The sample surfaces were analyzed by photoelectron spectroscopy using a W.A. Technology spectrometer with conventional monochromatized Al $K_{\alpha}$ source and ultra-high vacuum conditions. STM results were obtained using either a home-made apparatus or the commercial Beetle STM (Besocke Company) with spectroscopy facilities. STM investigations were done in air in the constant current mode, with typical tunneling current of 1-4 nA. Tips were obtained by electrochemical etching from Pt90-Ir10 alloy wires. Tunneling spectroscopy data were recorded between $-5 \mathrm{~V}$ and $+5 \mathrm{~V}$, and the method proposed by Feenstra et al. $[5,6]$ was applied to normalize all the measurements. 


\section{Results}

\subsection{X-ray photoelectron spectroscopy}

Figure 1 gives typical XPS spectra obtained from graphite after cleavage (a) and after oxidation with nitric acid (b).

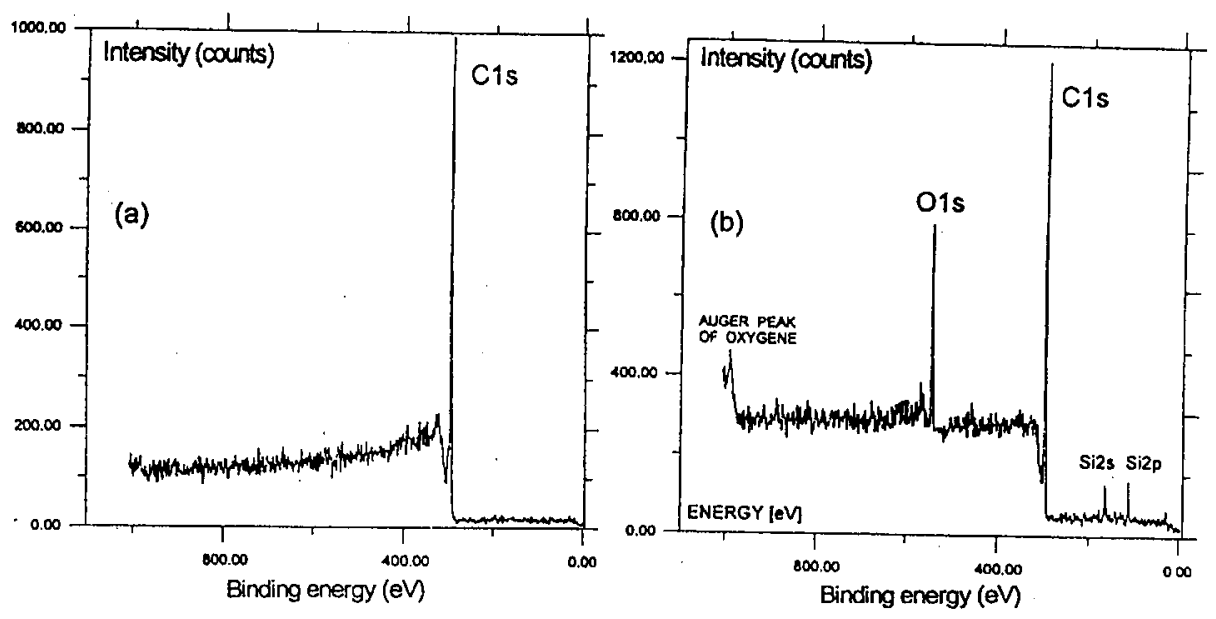

Fig. 1. XPS spectra obtained from a freshly cleaved HOPG surface before (a) and after (b) an oxidation treatment in $\mathrm{HNO}_{3} 4 \mathrm{~N}$.

The graphite surface is inert and no trace of oxygen contamination are visible from XPS. On the oxidized surface the O $1 s$ core level is well apparent. The Si $2 s$ and Si $2 p$ core levels are also detected. They are attributed to silica contamination coming from the beaker used for oxidation process. It is also noticeable on these wide range energy spectra that the satellite visible, in Fig. 1a, at around $20-30 \mathrm{eV}$ on the high binding energy side of the $\mathrm{C} 1 s$ photoelectron line, is strongly attenuated after oxidation (Fig. 1b). The satellite is typical of plasmon energy loss and its intensity decrease is a first indication that the valence band electrons which are involved in plasmon excitations have a different configuration after the oxidation treatment, in the thin surface layer probed by XPS.

The comparison of $\mathrm{C} 1 s$ photoelectron lines of graphite before and after oxidation is shown in Fig. 2.

Additional structures are clearly visible on the oxidized sample (Fig. 2b). These structures are well documented and are due to functionality groups created on the graphite surface by oxidation [3, 4]. One can also notice that the structure at $5.5 \mathrm{eV}$ on the high binding energy side of $\mathrm{C} 1 s$ peak is less intense after oxidation process.

That structure is attributed to $\pi \rightarrow \pi^{*}$ shake-up satellites and involves transitions between occupied and unoccupied $\pi$ bands of graphite. Its decrease is also a strong indication that valence and conduction bands have been markedly modified by oxidation. That modification of the surface electronic structure will be confirmed in the following by tunneling spectroscopy experiments. 


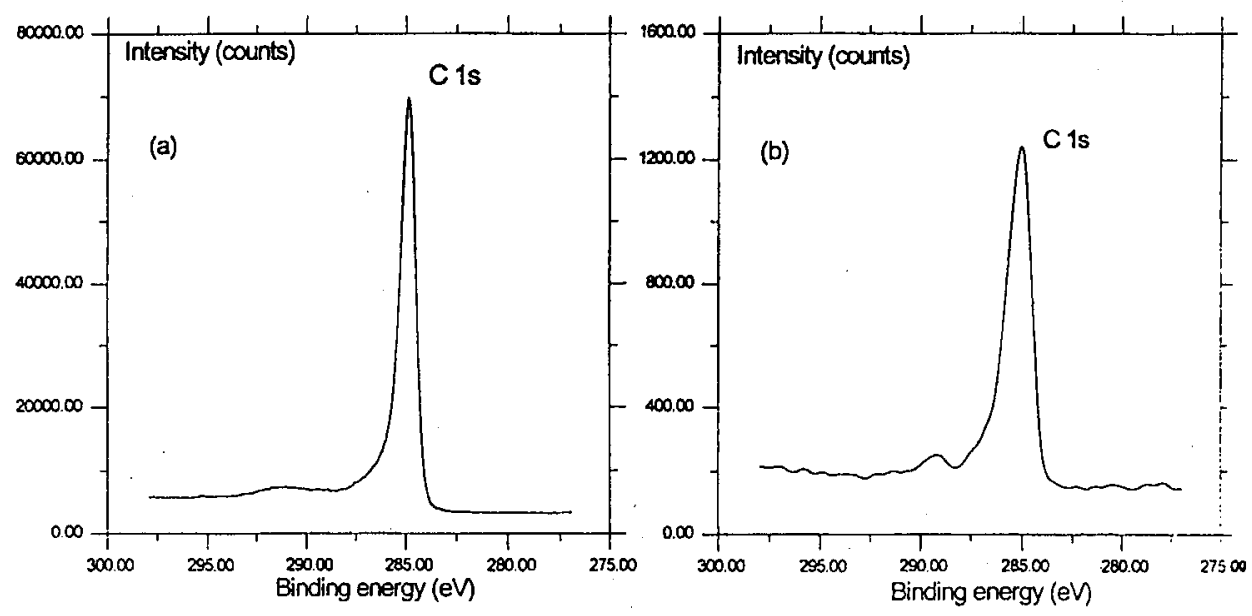

Fig. 2. XPS C $1 s$ peaks from (a) the pure HOPG surface and (b) the oxidized HOPG surface. The peak near $289.5 \mathrm{eV}$ on (b) is attributed to carboxylic groups.

\subsection{Scanning tunneling microscopy}

STM studies of oxidized samples confirm the important modification of the basal plane of graphite. Imaging on several samples (Table) and on several places for each sample gives evidence of two kinds of defects.

We first observed, in addition to the normal corrugation typical of the graphite surface, structures that are different from graphite. They have a general appearance of wavy structure with a small period around $0.25 \mathrm{~nm}$ close to the period in graphite and a larger period (Fig. 3). Even though we have not enough matter to interpret these images in details it is evident that the graphite surface has been modified and that carbon-carbon surface bonds have been opened to allow the creation of carboxylic surface groups. This corroborates the XPS results. STM images indicate moreover that the functionality groups are not randomly distributed but they arrange themselves in ordered fashion.

Secondly there are also places on the surface where tunneling current is quite unstable and where apparent defects are in the nanometer scale (Fig. 4). These areas should correspond to deeper oxidation of graphite. They are especially observed in the case of samples $C$ and $D$, but we also notice that samples $A, B$ and $E$ which were originally less oxidized present a quite similar surface after some days of exposition to ambient air.

One of the purpose of this work was to try to modify the graphite surface at atomic level. So far, the best conditions we found to create atomic modifications are those given for the sample $B$, i.e. 10 min oxidation at $70^{\circ} \mathrm{C}$ in $4 \mathrm{~N}$ nitric acid. Of course it seems very difficult to avoid any deep oxidation because defects initially present on the non-ideal HOPG surface, such as pits and steps, are more favorable sites for oxidation than the graphite basal plane can be. 


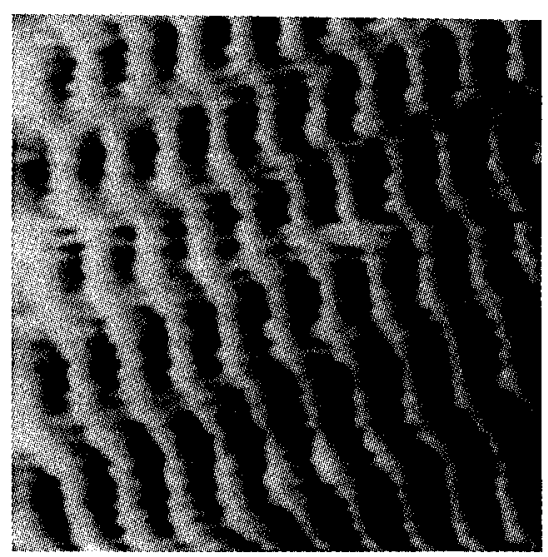

Fig. 3

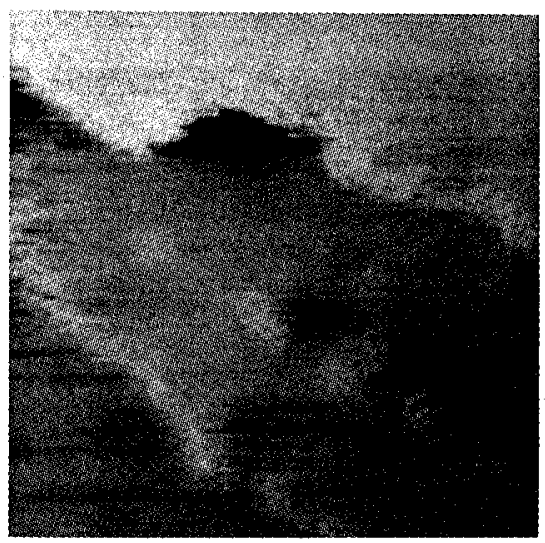

Fig. 4

Fig. 3. High resolution STM image of the waving form induced by oxidation on the graphitic lattice. Field of view is $2.6 \mathrm{~nm} \times 2.6 \mathrm{~nm}$. Tip biasing was $-100 \mathrm{mV}$, tunneling current $-7 \mathrm{nA}$.

Fig. 4. Large scale STM image illustrating deep oxidation effects: contamination on defects (on basal plane) and etching along the step. Field of view is $21 \mathrm{~nm} \times 21 \mathrm{~nm}$. Tip biasing was at $-100 \mathrm{mV}$, tunneling current $-1 \mathrm{nA}$.

\subsection{Scanning tunneling spectroscopy}

Figure 5 presents typical current-voltage curves recorded for pure graphite and oxidized graphite. Each curve is the average of 196 elementary curves taken over $2 \AA \times 2 \AA$ area. It is immediately apparent that the behavior for oxidized graphite is different, in particular at positive tip bias. Different recordings on the oxidized graphite also indicate local differences on that surface after oxidation.

Figures 6 and 7 present spectroscopic data for pure and oxidized graphite in the form $(\mathrm{d} I / \mathrm{d} V) /(I / V)$ versus tip bias which is expected to relate the local density of states $[5,6]$. On unoccupied spectrum from pure graphite surface characteristic features can be distinguished at $1.7 \mathrm{eV}$ and $2.2 \mathrm{eV}$ that can be attributed to specific points of the Brillouin zone $\left(P_{3}^{-}-Q_{2 \mathrm{~g}}^{-}\left(\pi_{2}\right)\right)$ and $\left(Q_{2 \mathrm{~g}}^{-}\left(\pi_{2}\right)\right)$, respectively (graphite band structure after Painter and Ellis [7]). Symmetry designations are based on the modified version quoted by Willis and Fitton [8]. Other structures at around $2.7 \mathrm{eV}$, noted $(+)$, and at $3.3 \mathrm{eV}$, noted IS, have been associated with an extrinsic surface state and to an interlayer state [9]. On the whole these results are in good agreement with experimental investigations by Reihl et al. [10] and by Fusch and Tosatti [11] or with theoretical work by Selloni et al. [12].

The spectra corresponding to the occupied states of pure graphite are subject to more variation than records from unoccupied states from point to point measurements. The main feature appears to be a maximum in the density of states lying at around 2-3 eV. It is attributed to flat $\pi$ band near $Q$ point in the Brillouin zone $\left(Q_{2 \mathrm{u}}^{-}\left(\pi_{1}\right)\right)$. Ultraviolet photoelectron spectroscopy data located also a feature coming from valence $\pi$ bands at $3 \mathrm{eV}$ below the Fermi level [13]. 


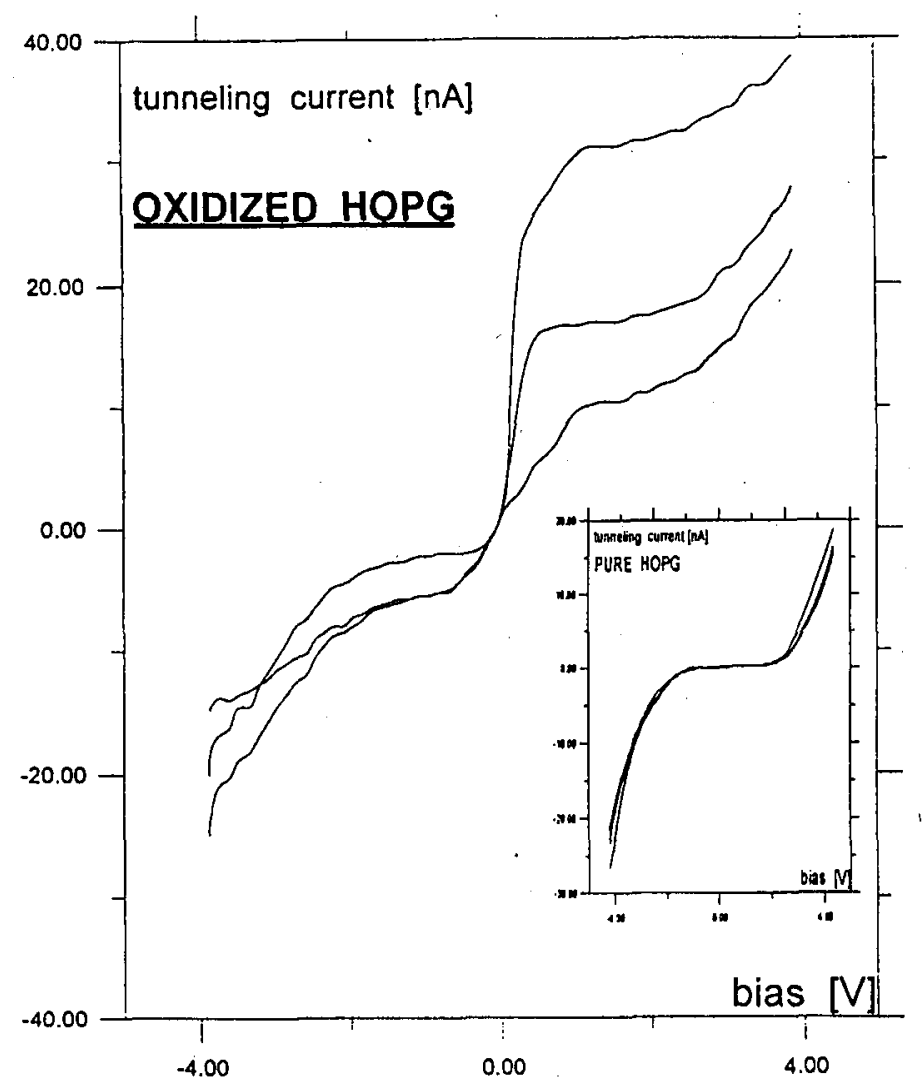

Fig. 5. Different current-voltage curves obtained on different places on oxidized HOPG samples. The inset shows typical results on pure graphite sample.

It is actually well apparent after Fig. 7 that spectroscopic data from oxidized surface differs markedly from the one from pure HOPG.

The main evolutions are the nearly complete vanishing of the structure around 1-2 eV, i.e. the bulk antibonding unoccupied states $\left(P_{3}^{-}-Q_{2 \mathrm{~g}}^{-}\left(\pi_{2}\right)\right)$ of mainly $\pi$ character in pure graphite have disappeared, and the relative emergence of the peak around $3-4 \mathrm{eV}$ that was attributed to an interlayer state.

The precise description of these spectroscopic data is not an easy task but we can however remark the coherence of the tunneling spectroscopy with the X-ray photoelectron spectroscopy results. The vanishing of $\pi$ bands for oxidized graphite is very well correlated with the decrease in the $\pi \rightarrow \pi^{*}$ shake-up satellite from $\mathrm{C} 1 s$ core level. The effect seems less pronounced for photoelectron spectroscopy because it is not a pure surface technic as tunneling spectroscopy. Of course the consequence of surface oxidation which has also been demonstrated by X-ray photoelectron spectroscopy is the creation of carboxylic groups on the surface. To create functional groups carbon-carbon bondings have to be broken before fixing oxygen or hydroxyl groups. Then $s p^{2}$ hybridization that describes pure graphite 
is no longer valid and $s p^{3}$-like bonds form. $\pi$ bands which form along due to $s p^{2}$ hybridization should also disappear when the graphite surface oxidizes.

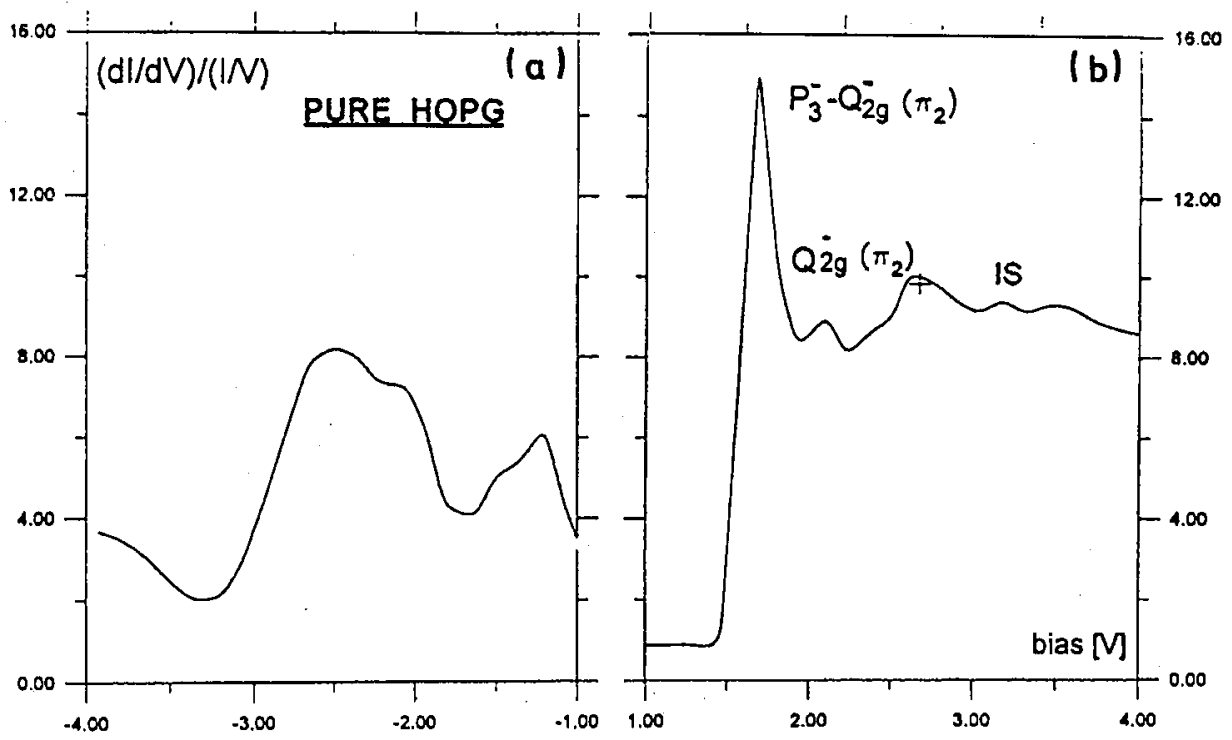

Fig. 6. Normalized tunneling spectroscopy STS data for occupied (a) and unoccupied (b) electronic states on pure HOPG surface.
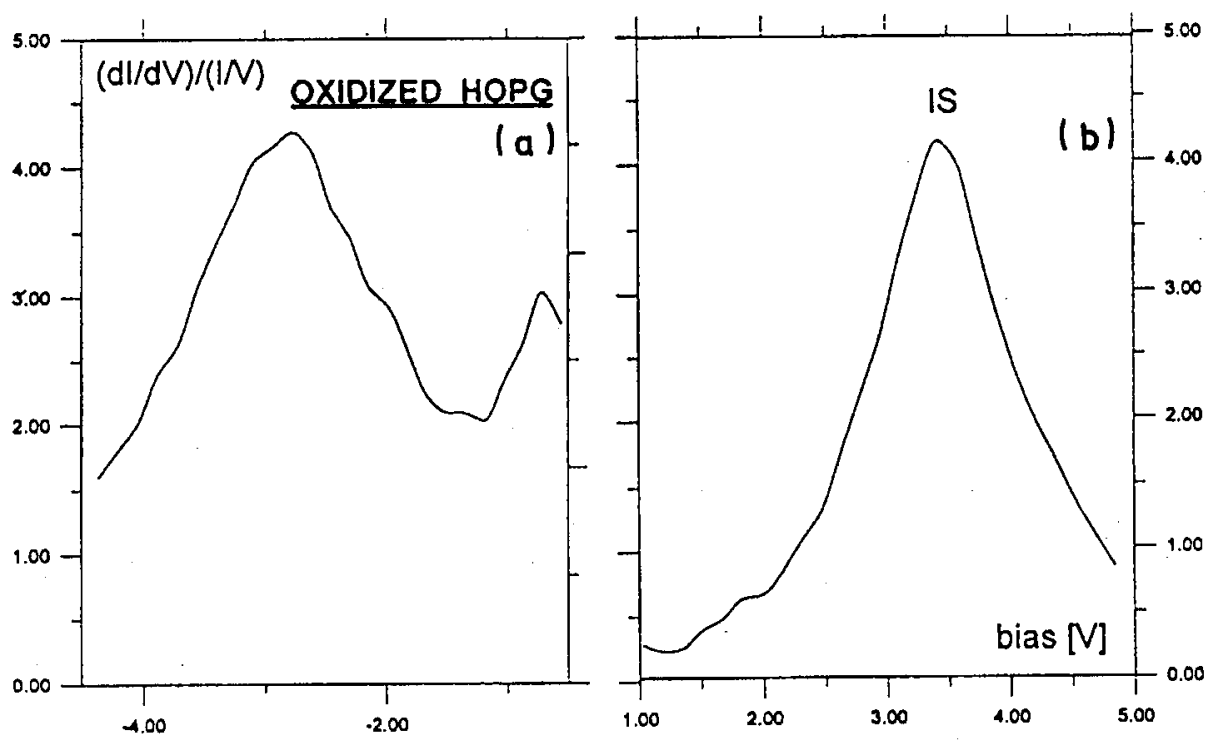

Fig. 7. Normalized tunneling spectroscopy STS data for occupied (a) and unoccupied (b) states on oxidized HOPG surface. 


\section{Conclusion}

Pure and oxidized graphite surfaces were studied using X-ray photoelectron spectroscopy and scanning tunneling microscopy and spectroscopy. XPS data demonstrated the effectiveness of the oxidation process and the creation of oxygenated surface groups on the basal plane of HOPG. The decrease in typical $\pi \rightarrow \pi^{*}$ shake-up transition on $\mathrm{C} 1 s$ photopeak is the indication that valence bands have been modified. The STM images of oxidized graphite revealed a well different atomic structure than the one typical from HOPG. It demonstrates that local atomic modifications have been produced on the basal plane.

Tunneling spectroscopy data confirm the view that $\pi$ bands vanish after oxidation due to $s p^{2}$ dehybridization along with the creation of functional oxidized surface groups.

This work emphasizes the complementarity of X-ray photoelectron spectroscopy and scanning tunneling microscopy-spectroscopy in studies of surface reactivity and functionalization.

\section{Acknowledgments}

We thank Marie-Paule Besland and Evelyne Bergignat for the X-ray photoelectron spectroscopy measurements. Z.K. is indebted to the Ministère des Affaires Etrangères Français for a grant during his stay at the Ecole Centrale de Lyon.

\section{References}

[1] L. Porte, D. Richard, P. Gallezot, J. Microsc. 152, 515 (1988).

[2] F. Atamny, R. Schlogl, W.J. Wirth, J. Stephan, Ultramicroscopy 42-44, 660 (1992).

[3] J.S. Hubacek, R.T. Brockenbrough, J. Microsc. 152, 221 (1988).

[4] A. Sartre, M. Phaner, L. Porte, G.N. Sauvion, Appl. Surf. Sci. 70/71, 402 (1993).

[5] R.M. Feenstra, A.P. Fein, Phys. Rev. B 32, 1394 (1985).

[6] R.M. Feenstra, J.A. Stroscio, A.P. Fein, Surf. Sci. 181, 295 (1987).

[7] G.S. Painter, D.E. Ellis, Phys. Rev. B 1, 4747 (1970).

[8] R.F. Willis, B. Fitton, J. Vac. Sci. Technol. 9, 651 (1972).

[9] Scanning Tunneling Microscopy I, Springer Series in Surface Physics, Eds. H.J. Guntherodt, R. Wiesendanger, Vol. 20, Springer-Verlag, Berlin 1992.

[10] B. Reihl, J.K. Gimzewski, J.M. Nicholls, E. Tosatti, Phys. Rev. B 33, 5770 (1986).

[11] H. Fusch, E. Tosatti, Europhys. Lett. 3, 745 (1987).

[12] A. Selloni, P. Carnevali, E. Tosatti, C.D. Chen, Phys. Rev. B 31, 2602 (1985).

[13] F.R. McFeely, S.P. Kowalczyk, L. Ley, R.G. Cavell, R.A. Pollak, D.A. Shirley, Phys. Rev. B 9, 5268 (1974). 